
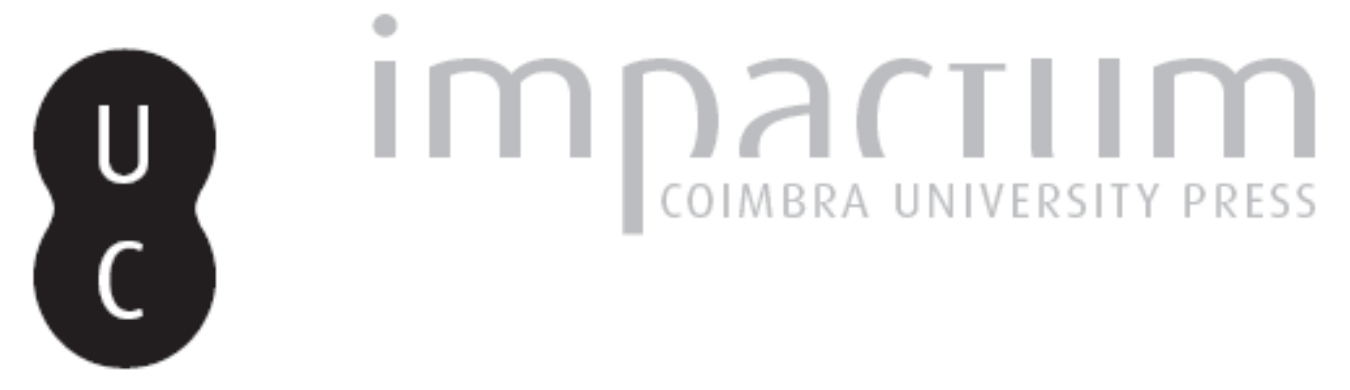

\title{
Insegnare italiano nelle scuole di musica
}

\section{Autor(es): $\quad$ Sacco, Marcello}

Publicado por: Imprensa da Universidade de Coimbra

URL persistente:

URI:http://hdl.handle.net/10316.2/42521

DOI:

DOl:https://doi.org/10.14195/0870-8584_5_8

Accessed : $\quad$ 26-Apr-2023 09:21:05

A navegação consulta e descarregamento dos títulos inseridos nas Bibliotecas Digitais UC Digitalis, UC Pombalina e UC Impactum, pressupõem a aceitação plena e sem reservas dos Termos e Condições de Uso destas Bibliotecas Digitais, disponíveis em https://digitalis.uc.pt/pt-pt/termos.

Conforme exposto nos referidos Termos e Condições de Uso, o descarregamento de títulos de acesso restrito requer uma licença válida de autorização devendo o utilizador aceder ao(s) documento(s) a partir de um endereço de IP da instituição detentora da supramencionada licença.

Ao utilizador é apenas permitido o descarregamento para uso pessoal, pelo que o emprego do(s) título(s) descarregado(s) para outro fim, designadamente comercial, carece de autorização do respetivo autor ou editor da obra.

Na medida em que todas as obras da UC Digitalis se encontram protegidas pelo Código do Direito de Autor e Direitos Conexos e demais legislação aplicável, toda a cópia, parcial ou total, deste documento, nos casos em que é legalmente admitida, deverá conter ou fazer-se acompanhar por este aviso.

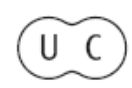




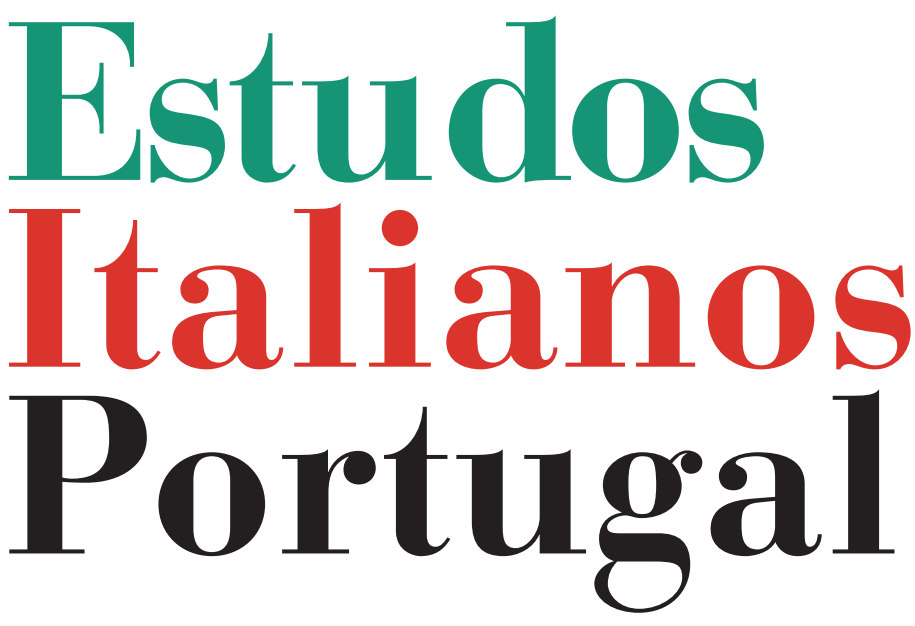

Instituto

Italiano

de Cultura

de Lisboa

Nova Série

$\mathrm{N}^{\mathbf{0}} 5$ 


\title{
INSEGNARE ITALIANO NELLE SCUOLE DI MUSICA
}

\author{
Marcello Sacco
}

Non esiste, che io sappia, un censimento esaustivo, ma è praticamente certo che ogni anno, in tutto il mondo, un numero presumibilmente elevato di stranieri consuma il primo approccio con la lingua italiana attraverso il repertorio operistico.

Al Conservatorio di Lisbona, per esempio, nell'anno scolastico appena trascorso $(2009 / 10)$ gli studenti di italiano erano circa una sessantina, trenta al Conservatorio di Oporto, 27 a Coimbra ${ }^{1}$. Va detto che il numero degli iscritti all'inizio dell'anno finisce per ridursi sempre un po' lungo il percorso. Il motivo di questo tasso di abbandono, che si aggira attorno al $20 \%$, si deve quasi sempre a incompatibilità di orari, in quanto si ha a che fare soprattutto con studenti giovani adulti, quasi tutti con percorsi universitari o professionali paralleli da conciliare con lo studio della musica (per motivi fisiologici, al

* Dopo il Master in "Estudos Portugueses" presso la Universidade Autónoma di Lisbona, ha di recente concluso il Master ITALS in Didattica e Promozione della Lingua e Cultura Italiane a Stranieri, presso l'Università Ca' Foscari di Venezia. È docente di italiano presso i corsi di canto del Conservatorio di Lisbona, Academia de Amadores de Música e Academia de Santa Cecília. Ha curato e tradotto diverse opere di autori portoghesi in Italia, collabora al blog letterario Sul romanzo (http://sulromanzo.blogspot.com/) e cura la rubrica online Lirici E lyrics dedicata ai testi di arie d'opera e canzoni (http://www.iltaccoditalia. info/sito/index.asp?s=4\&t $=54)$.

1 Ringrazio la prof.ssa Maria Pia Mottini, del Conservatorio di Oporto, e la prof.ssa Elisabete Mira, del Conservatorio di Coimbra, per le informazioni relative ai rispettivi istituti. 
contrario di quanto avviene con gli altri strumenti musicali, gli allievi di canto lirico non sono ammessi al corso prima dei $16 / 17$ anni e una buona parte di essi ha tra i 20 e i 30 anni).

Più in dettaglio, gli allievi di italiano dei conservatori portoghesi frequentano un corso di durata triennale e seguono il consueto calendario scolastico da fine settembre ai primi di giugno. A Lisbona sono divisi in sei classi, le cui lezioni sono distribuite in due "blocchi" didattici settimanali consecutivi, ciascuno dei quali di 45 minuti (dunque sostanzialmente una lezione settimanale di 90 minuti). Anche Coimbra organizza il corso in due blocchi di 45 minuti, ma ha trovato didatticamente più proficuo dividerli in due incontri settimanali. Lo stesso numero di ore settimanali non è cosa del tutto scontata, poiché l'autonomia pedagogica di ciascun conservatorio ha finora permesso che la situazione variasse a seconda delle città. Per questo a Oporto i blocchi didattici settimanali sono stati fino all'anno scorso tre, dunque 135 minuti da dividersi in due incontri settimanali. Lo stesso vale per l'esistenza di un esame al termine del terzo anno; esame che a Oporto è obbligatorio, ma non a Lisbona e Coimbra, dove si presentano a tale esame solo allievi autoproposti, anche esterni, che non abbiano regolarmente frequentato il corso o che lo abbiano frequentato con risultati negativi. A questa mancanza di omogeneità, non necessariamente deprecabile, nell'applicazione di leggi e regolamenti va aggiunto un tale fervore legislativo degli ultimi anni che le informazioni riportate qui e ora potrebbero risultare obsolete o superate gia nei prossimi mesi.

Facendo ora un po' di storia, e stando a quanto riportato da altri autori ${ }^{2}$, l'insegnamento dell'italiano nel conservatorio

2 Cfr. J. P. Alvarenga (coord.), João Domingos Bomtempo (catalogo dell'esposizione omonima), Lisboa, Instituto da Biblioteca Nacional e do Livro, 1993; L. Mioni, "Cantar em italiano e imaginar fazê-lo. A pronúncia do italiano para estudantes de canto lírico", Imaginação e literatura, coordenação de Rita Marnoto, Coimbra, Instituto de Estudos Italianos da FLUC, 2009, pp. 121-136; M. J. Borges, "Historial" em http://www.em-conservatorio-nacional.com/ 
di Lisbona esiste sin dalla sua fondazione, a opera di João Domingos Bomtempo, nel 1834. Il dato è particolarmente interessante, perché fa di tale cattedra la più antica fra quelle attive oggi in Portogallo ${ }^{3}$, mentre a Oporto l'insegnamento della lingua italiana era già incluso nel programma ufficiale pubblicato sul Diário do Governo del 10-3-1919. Più recentemente, il decreto ministeriale n. ${ }^{\circ} 76$ del 9 ottobre 1985 fa menzione di due ore di italiano obbligatorie accanto alle altre materie necessarie al completamento del corso di Canto. $\mathrm{Si}$ aggiunga che, oltre alle cattedre citate, altre dello stesso genere sono attive presso i conservatori di Aveiro e Braga, mentre scuole come l'ultracentenaria Academia de Amadores de Música di Lisbona o la prestigiosa Academia de Santa Cecília di Ameixoeira, a cui il Ministero riconosce il cosiddetto "paralelismo pedagógico", seguono gli stessi programmi, sia pur con un numero ben più ridotto di allievi e continuità a singhiozzo, secondo la domanda del mercato delle iscrizioni (sei iscritti presso la Academia de Amadores de Música e solo due allieve di secondo anno presso la Academia de Santa Cecília per l'anno scolastico 2009-2010).

Se a tutti questi studenti aggiungiamo la folla dei melomani e se pensiamo che, come vuole un vecchio luogo comune tutt'altro che lontano dal vero, non passa giorno senza che almeno un teatro al mondo metta in scena un'opera di Verdi (e abbiamo citato solo Verdi), risulta persino evidente che la nostra lingua gode oggi di una proiezione internazionale garantita da autori che non siamo abituati ad annoverare nei nostri canoni letterari. Con una battuta, si potrebbe dire

3 Mi piace inoltre segnalare che, negli ultimi anni, le cattedre sia di italiano che di tedesco del Conservatorio di Lisbona hanno dato alla luce due testi pionieristici nell'ambito tanto dell'insegnamento rivolto a cantanti come dell'insegnamento in chiave contrastiva con la L1 degli studenti destinatari (il portoghese). Mi riferisco al dizionario di Leonor Lucena, Dicionário do Cantor Lírico - Italiano/Português, Lisboa, 2009, e al manuale di Barbara Schilling Tengarrinha, Deutsch für Sänger/Alemão para cantores, Norderstedt, BOD, 2008. 
che siamo forse davanti a uno scherzo giocato al moderno dal postmoderno, perché siamo alla paradossale rivincita dei Piave sui Dante, checché ne dicano i cultori della scrematura dei canoni. E guardando oltre la diffusione meramente scolastica o teatrale, con un occhio ai nuovi mezzi di diffusione audiovisiva, come non vedere che un DVD edito da una multinazionale, magari con i suoi spezzoni immancabilmente inseriti in youtube, conferisce ai versi non sempre eccelsi della Traviata una capacità di penetrazione domestica mondiale (per di più nell'originale italiano) che difficilmente un'ottima traduzione dei Canti leopardiani potrà eguagliare? Uno spunto sul quale gli glottodidatti dovrebbero forse riflettere più a fondo. 